\title{
EDITORIAL
}

(O) BIOLOGIC THERAPY FOR IBD

\section{The expanding role of biologic therapy for IBD}

T he topic of biologic therapy for IBD has been selected for this Focus issue of Nature Reviews Gastroenterology \& Hepatology because of the important implications it has for disease management. Four biologic agents are currently marketed for the treatment of IBD in the US and around the world, three of which target tumor necrosis factor (TNF). When considering the impact of biologic therapy on the management of IBD, it is important to consider the positioning of the biologic agents as well as evidence of their safety, efficacy and their impact on quality of life, disability and pharmacoeconomics.

Crohn's disease and (to a lesser extent) ulcerative colitis are chronic, progressive, immune-mediated inflammatory disorders that comprise an overlapping spectrum of mucosal, transmural and extraintestinal manifestations. Whether Crohn's disease and ulcerative colitis are actually two ends of a spectrum or are unique disease entities remains controversial. However, the increased incidence of these conditions within families and the potential for 'phenotypic shiff' during the disease course, or after bowel surgery, suggest that they represent phenotypic manifestations of similar genetic disorders driven by environmental triggers. 'Pathophysiological morphing' of disease can also occur, in which patients become unresponsive to an initially efficacious therapy; for instance patients who initially respond to anti-TNF therapy can subsequently redevelop mucosal ulcerations despite the presence of adequate concentrations of therapeutic monoclonal antibodies. As we continue to dissect the immune and inflammatory pathways leading to heterogeneous phenotypic manifestations, we may progress towards the development of targeted treatments for patients with specific pathophysiologic or immuno-inflammatory subtypes.

Strong evidence exists for the efficacy of some biologic agents for the treatment of Crohn's disease and ulcerative colitis, but even among agents that target TNF, differential responses occur in various disease settings. For the $\mathrm{IgG}_{1}$ monoclonal antibodies infliximab and adalimumab, and certolizumab pegol, the pegylated Fab' fragment of humanized anti-TNF monoclonal antibody, there is strong evidence of efficacy in patients with Crohn's disease and rheumatoid arthritis, and yet, etanercept, a TNF receptor antagonist that is effective in patients with rheumatoid arthritis, has not been beneficial for the treatment of Crohn's disease. Hence, improved means of identifying and predicting which patients are most likely to respond to individual or specific combination therapies are clearly needed.

The ability to identify which patients are most likely to benefit-and I use the term to mean more than simply respond-is a pervasive theme throughout all of the accompanying articles in this issue. The heterogeneity of patients with IBD in terms of disease extent and severity, extraintestinal manifestations and therapeutic responsiveness is a considerable obstacle to the development of recommendations for optimal treatment. For example, approximately $50 \%$ of patients with ulcerative colitis or Crohn's disease never require corticosteroid therapy. However, at the other extreme, in patients who do require steroids, the long-term development of dependency or refractoriness to steroids is more the rule than the exception. Furthermore, although Crohn's disease is a progressive transmural disease, intuitively, we can expect that the likelihood of response to any therapeutic strategy is greater for patients treated before the development of fibrotic stenosis or significant fistulization. This expectation has been borne out in studies with thiopurines and biologic agents, as discussed by D'Haens on page 86 of this issue, and provides a rationale for a top-down (early introduction of aggressive treatment) approach, including immunosuppressives or biologic agents once corticosteroids are indicated, to induce clinical remissions. Factors such as extensive disease (particularly in children), transmural complications at diagnosis, perianal disease, deep mucosal ulcerations, cigarette smoking, early need for corticosteroids and high titers of serological markers identify patients at risk of developing 'progressive' Crohn's disease (frequent courses of steroids or the need for surgery). However, as yet, no phenotypic indicators or biomarkers can predict a patient's response to any specific therapy. This would be the penultimate 'holy grail' for IBD therapy and would be trumped only by the identification of cause(s) and potential cure(s) for these chronic, currently-incurable, immune-mediated inflammatory diseases.

A further impediment to predicting an individual's disease course is the lack of validated measures of structural damage as a potential end point for assessing disease modification in Crohn's disease. On page 79, Van Assche and colleagues discuss the concept of 'disease modification' in Crohn's disease and current surrogate markers, such as reductions in the number of hospitalizations and surgeries. By contrast, our rheumatology colleagues have the ability to assess joint-space narrowing and erosions on

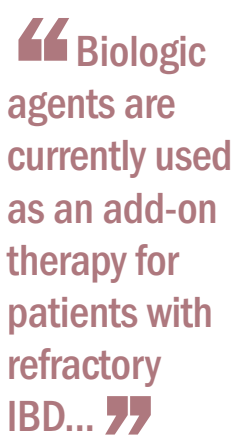

Stephen B. Hanauer is the Editor-in-Chief of Nature Reviews Gastroenterology \& Hepatology.

Competing interests The author declares associations with the following companies: Abbott Labs, Alevan, Amgen, Astellas

Pharma Global, Astra Zeneca, Bristol Myers Squibb, Centocor, Chemocentryx, Elan, Ferring, Genentech, GSK, McNeil PPC, Millennium

Pharmaceuticals, Novartis, Pfizer, Prometheus, Salix, Shire, Takeda, Therakos, UCB Pharma and Warner-Chilcott. See the article online for full details of the relationships. 


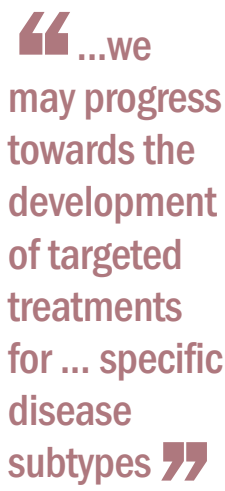

X-rays of a patient's hands, which enabled researchers to observe how DMARD (disease-modifying antirheumatic drug) therapies can alter the 'natural history' of rheumatoid arthritis. Indeed, even the measure of structural damage of joints, while a validated and reproducible quantifiable measure, does not sufficiently reflect disease burden that accounts for disability (Yazici. Y, Sokka, T. \& Pincus, T. Rheum. Dis. Clin. North Am. 35, 723-729, vi; 2009). Nevertheless, the impact of early intervention on a progressive structural disease process (similar to transmural disease complications of Crohn's disease) demonstrates that anti-TNF and other biologic therapies prevent the development of structural damage when they are employed before joint (or bowel wall) damage occurs. The absence of such a gauge of structural damage and quantitative end point in IBD has led to the use of changes in mucosal healing, surgeries and hospitalizations as surrogates for disease modification-instruments such as the Crohn's Disease Activity Index (CDAI) and ulcerative colitis disease activity indices reflect disease symptoms more than they do inflammatory or structural burden.

Biologic agents are currently used as an add-on therapy for patients with refractory IBD not responding to aminosalicylates, corticosteroids and/or immunosuppressants. Hence, the safety of these agents must be considered with respect to that of concomitant medications. While these therapies clearly increase the potential for infectious complications, particularly with regard to opportunistic infections as de Silva and colleagues discuss on page 93 of this issue, the majority occur in patients receiving corticosteroids or multiple immunosuppressive agents. In the setting of early rheumatoid arthritis, when anti-TNF agents are used without corticosteroids or other immunosuppressives, their adverse effects are markedly attenuated compared with their use in patients who have later stages of disease or are on concomitant immunosuppressives. Similarly, in patients with extensive or long-duration IBD the risk of lymphomas associated with biologic anti-TNF agents is difficult to isolate from that conferred by concurrent use of thiopurines; the latter predispose patients to EpsteinBarr virus-associated lymphoproliferative disorders and lymphomas in the absence of anti-TNF exposure. Thus, the early use of biologic agents in the treatment of patients with IBD may result in a substantial improvement in the risk of infections and, possibly, lymphomas. Regardless of the increase in the relative risk of infections and neoplasia associated with the use of biologic agents, the absolute risk remains very low despite the obfuscation of concomitant therapies.

The benefit of biologic therapies for IBD also requires consideration of the comparative effectiveness and pharmacoeconomics of these medications; neither factor, as yet, has been assessed in clinical trials. The main entry point for these agents into the marketplace has been to treat refractory disease as demonstrated by trials that randomly allocate responders (those who experience symptomatic improvement) to maintenance therapy with the same biologic therapy, or placebo. Since these agents are efficacious in populations of patients who have not responded to conventional therapies, a reasonable assumption is that biologic therapies (both anti-TNF agents and natalizumab) are the most effective therapies for IBD. However, we have yet to perform adequate comparative effectiveness trials in patients with early disease to demonstrate that top-down therapy with a biologic agent is more effective, less risky or more cost effective than conventional therapies in the short term or long term. As Cohen also points out on page 103 of this issue, we need appropriately designed and timely pharmacoeconomic studies to assess both symptomatic end points and total health-care-related costs, including those of medications, visits, procedures, hospitalizations and surgeries. He also advocates the assessment of indirect costs pertaining to loss of occupation, spouse and family interruptions in income related to family illness, and ultimately disability.

We are confronted with a spectrum of idiopathic IBD without known medical cures. Substantial inroads have been made into the short-term and long-term management of Crohn's disease and ulcerative colitis such that their associated mortality is minimal compared with that of the general population. Nevertheless, these diseases present substantial personal and societal burdens. Unraveling the immune and inflammatory pathways associated with IBD has led to biologic agents that specifically inhibit TNF signaling, and target adhesion molecules, which are involved in IBD and other chronic, immune-mediated inflammatory disorders. Yet, as one would predict, and as Melmed and Targan discuss on page 110 of this issue, no single therapy is efficacious for all afflicted individuals. As the pathogenesis of these diseases continues to be elucidated we must continue to seek out panels of genetic, immune and environmental attributes to aid our prediction of responses to specific therapeutic targets. Already, as investigations of immune system-microbial interactions, delineation of environmental triggers (for example, the dichotomous impact of cigarettes on the course of ulcerative colitis and Crohn's disease), and studies of proteomics and metabolomics advance, one would readily anticipate identification of novel therapeutic targets for biologic and conventional therapies. Optimal use of these evolving therapies will require identification of specific populations of patients who are most likely to benefit from specific interventions. Ultimately, the targets of biologic therapies should be amenable to the development of additional cost-effective, conventional treatment approaches.

doi:10.1038/nrgastro.2009.238 